\title{
Exploring the 4th dimension: hippocampus, time, and memory revisited
}

\author{
Bin Yin ${ }^{1 *}$ and Andrew B. Troger ${ }^{2}$ \\ Department of Psychology and Neuroscience, Duke University, Durham, NC, USA \\ 2 Department of Neurobiology, Harvard University, Cambridge, MA, USA \\ *Correspondence: bin.yin@duke.edu
}

Accurate and reliable timing is an essential component of nearly every purposeful behavior. Just as the brain contains mechanisms to track and orient the body in space, so too must it be able to orient itself in time. Coincidence detection - the integration of simultaneous activation of multiple inputs - is a proposed solution to the question of how the brain tracks the duration of events in the seconds-to-minutes range using millisecond-scale neural processes (Matell and Meck, 2000). The striatal beat-frequency (SBF) model is one of the most successful attempts at explaining the neural basis of interval timing in terms of coincidence detection of oscillatory processes (Matell and Meck, 2004; Lustig et al., 2005; Harrington et al., 2010; Oprisan and Buhusi, 2011, submitted). The SBF model involves a set of cortical timekeeper neurons that oscillate at regular, but distinct frequencies, allowing a unique pattern of activation to occur at each point in time. These activation patterns project onto striatal integrators that combine their information with feedback (e.g., reward input) and form the basis of interval timing.

Independent lines of research appear to converge on the conclusion that functional circuits composed of the prefrontal cortex, striatum, and thalamus are instrumental to both time perception and timed performance (Coull et al., 2004, 2011; Hinton and Meck, 2004; Buhusi and Meck, 2005; Meck, 2006a,b; Yin, 2009; Allman and Meck, 2011). This frontal-striatal system is hypothesized to correspond to the functional components of the SBF model (Meck, 1996, 2006a,b; Meck and Benson, 2002; Matell et al., 2003; Matell and Meck, 2004; Meck et al., 2008), wherein cortical oscillatory neurons, and reward input from the substantia nigra are integrated by striatal medium spiny neurons (MSNs). These neurons can hold temporal "memories" via dopamine-facilitated long-term potentiation and long-term depression that, possibly via $\alpha$-amino-3-hydroxy5-methyl-4-isoxazolepropionic acid receptor (AMPAR) trafficking (Centonze et al., 2001), modulate synaptic weights. Later, when the same signal duration is timed again, these neurons compare the current pattern of cortical activation with the stored "memories"; if coincidence is detected, then the spiny neurons fire to indicate the target duration has elapsed.

These neural structures contained within cortico-striatal circuits may not be the only ones involved in interval timing, however. The role of the hippocampus in timing and time perception for durations in the supraseconds range was initially explored by Meck et al. (1984). Since then, numerous studies have demonstrated reliable changes in the accuracy and precision of interval timing following a variety of techniques impacting hippocampal function (e.g., transection of the fimbria fornix, lesions of the medial septal area, resection of the temporal lobe, selective lesions of the dorsal hippocampus, and destruction of the entire hippocampus - see Balci et al., 2009 for a review). Nevertheless, an explanation of the effects of hippocampal damage within the context of a theoretical model of interval timing has been elusive (Grossberg and Merrill, 1992, 1996; Lytton and Lipton, 1999; Onoda et al., 2003; Matell and Meck, 2004; Sakata, 2006; Lewis et al., 2011). As a consequence, the primary goal of this opinion article is to outline mechanisms by which the hippocampus could have specific effects on the modulation of the neural circuits specified by the SBF model of interval timing.

Rats and mice with lesions of the hippocampus and related areas demonstrate a proportional "leftward" shift in distributions of timing judgments for intervals in the range of 2-8 s for temporal bisection procedures and 10-40 s for peak-interval timing procedures - that is, when faced with tasks requiring them to estimate or reproduce a specific duration, they respond earlier on average than normal subjects indicating an over estimation/under production of duration proportional to the anchor durations or target duration(s) being timed (Meck et al., 1984, 1987; Olton et al., 1987, 1988; Buhusi et al., 2004; Balci et al., 2009). Similar effects on timing have also been observed in human participants with hippocampal damage following temporal lobe resection for anchor durations spanning the ranges of 50 vs. $200 \mathrm{~ms}, 1$ vs. $2 \mathrm{~s}$, and 2 vs. $8 \mathrm{~s}$ in temporal bisection procedures and $0.5-8 \mathrm{~s}$ for temporal reproduction procedures (Vidalaki et al., 1999; Melgire et al., 2005). Interestingly, in both rodents and humans, an increase in the precision of timing often accompanies the distortion in the accuracy of the temporal representations (Meck et al., 1984; Vidalaki et al., 1999; Meck, 2002, 2005; Melgire et al., 2005). These "classic" effects of hippocampal lesions on the performance of rats in the peak-interval procedure are illustrated in Figure 1.

Though there have been a number of studies that suggest a lack of any effect on peak-interval timing procedures in hippocampally lesioned animals (Dietrich et al., 1997; Dietrich and Allen, 1998), these experiments included extensive post-lesion training with explicit reinforcement contingencies for probe trials. Evidence suggests that, with extensive training, it is possible for timing behavior to become habitual and to enter a "locked" state where the "classic" horizontal shifts of response functions to pharmacological challenges are no longer apparent (Yin and Knowlton, 2006; Cheng et al., 2007a,b; Yin et al., 2009). It is also known that in cases of extensive training, hippocampal function can be transferred to other brain areas such as the cortex (Wiltgen and Silva, 2007; Wiltgen et al., 2010).

There are several important roles that the hippocampus could play in the SBF timing circuit. Firstly, it could function as a feedback control mediator (Meck, 1988), participating in the determination of temporal expectancy, 


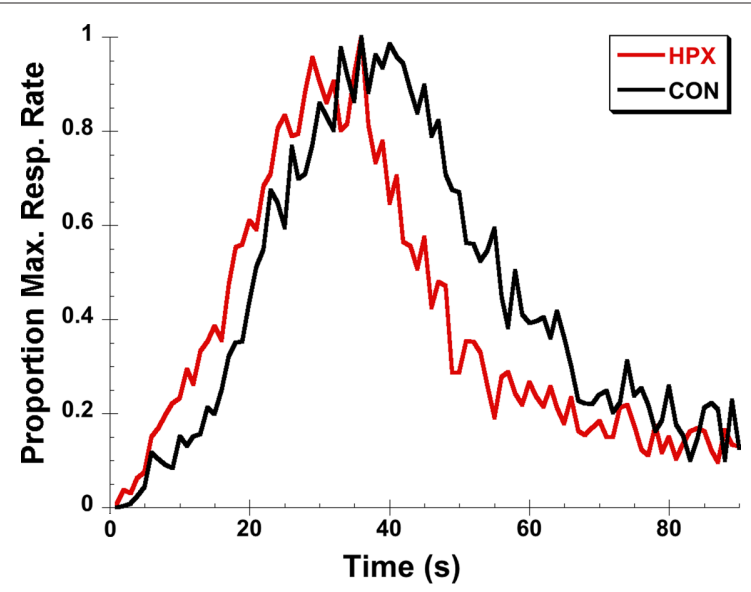

FIGURE 1 |An illustration of the "classic" effects of post-training hippocampal lesions for rats trained on a 40-s peak-interval procedure. The peak functions of rats with hippocampal lesions (HPX) rats are shifted leftward relative to control (CON) rats and are sharper with less spread around the observed peak time. Data are replotted from Buhusi et al. (2004).

which is a continuously updated function of memory, and clock-reading that supports the anticipation of outcomes tied to specific durations. Separate cortical areas exist that participate in the cortico-striatal and frontohippocampal circuits, respectively. The former is the basis of the "clock" stage while the latter may modulate the "memory" stage, updating temporal expectancy on a trial-bytrial basis. This memory-modulating cortical area also sends input to the striatal MSNs. Given that hippocampal lesions produce a progressive leftward shift (under production/over estimation) and frontal lesions produce a more or less symmetrical progressive rightward shift (over production/under estimation), it is possible that the hippocampus works in tandem with this frontal-temporal regulatory circuit to update temporal expectancy on a trial-by-trial basis (Meck et al., 1987; MacDonald and Meck, 2004; Lustig et al., 2005).

A second function that the hippocampus might serve in timing and time perception is as a regulator of the dynamic firing threshold of striatal MSNs (Matell and Meck, 2004). Hippocampal-striatal interactions have been previously documented (Devan and White, 1999; Poldrack and Packard, 2003; Lee et al., 2008; Graham et al., 2009). The MSN is essentially a two-state system with a "down-state" that does not allow neural firing and an "up-state" that facilitates firing. State transitions are driven by excitatory inputs. The interspike interval varies because the sub-threshold membrane potential fluctuates (Stern et al., 1997).
Properties of sub-threshold signal integration in MSNs are determined by the distribution of synaptic inputs and differential activation of multiple postsynaptic conductances (Carter et al., 2007).

On this basis, we can suggest two possible ways that hippocampal input could directly contribute to modulating striatal neuron firing: phasic excitation and tonic inhibition. The hippocampus could desensitize membrane AMPARs on MSNs with its phasic excitatory output when it detects minor environmental changes, such as at the beginning of a new "to be timed" signal. This would render a varying set of MSNs unable to use "memories" of the previous signal duration. These MSNs must then update their "memories" on a trial-by-trial basis. This would produce more trial-by-trial variation, and would be expected to contribute to the Gaussianlike noise that generates scalar timing (see Matell and Meck, 2004; Oprisan and Buhusi, 2011, submitted).

The hippocampus could also tonically inhibit, and thus lower the sub-threshold membrane potential of striatal neurons such that firing is delayed by a small duration in some proportion of MSNs. Such an effect would be more pronounced in heavily weighted synapses of MSNs corresponding with the "representation" of the previous trial's temporal sequence of responding and reward outcome. In this case, striatal neurons could display "overexcitement" in the absence of hippocampal inhibition followed by habituation, resulting in a leftward shift of the timing function in early trials followed by a return to a more normal response distribution following repeated testing, again possibly explaining the lack of an observed shift in lesioned animals with extensive training.

A third possibility is that the hippocampus might function in a downstream decision-making process that controls motor output. It has been suggested that the decision-making processes downstream of the "clock stage" deserve further investigation (Harrington et al., 2004, 2011; Wearden, 2004; Meck, 2005). A subject's selection and execution of motor action based on the clock's output (which in the SBF model is determined by striatal firing rates) may depend on a "threshold gating" mechanism located in another brain region (Gibbon et al., 1997; Jin et al., 2009; Höhn, et al., 2011). This would predict variation in timing behavior between subjects that have identical perceptions of duration. For example, in a peak-interval procedure, an "impulsive" subject may press the lever well before its perception of the time in the current trial matches a sample taken from its memory distribution of times of reinforcement on previous trials. Conversely, a "less impulsive" subject demonstrating a higher degree of "self control" may be reluctant to press a lever until the time on the current time is much closer to the remembered target duration - or even past this duration (Church et al., 1994).

Regions that might be involved in this subsequent action-selection process are the ventral and dorsomedial striatum, orbitofrontal cortex, and possibly the hippocampus (Johnson et al., 2007; MacDonald et al., 2011). Indeed, it has been reported that the hippocampus may have a role in controlling impulsivity (Cheung and Cardinal, 2005; McHugh et. al., 2008; Sala et al., 2011). On the other hand, it has been shown that ventral/medial striatal neurons are entrained to the hippocampal theta rhythm (Berke et al., 2004). Therefore, it seems reasonable to speculate that the hippocampus might interfere with the downstream temporal control of action sequences (most likely via inhibitory control) in tandem with the ventral/medial striatal neurons. Lesions of the hippocampus may diminish this inhibitory control, thereby resulting in earlier start times, leading to leftward horizontal 
shifts of the peak function in the peakinterval procedure (Meck et al., 1984, 1987; Balci et al., 2009; MacDonald et al., 2011). These three possibilities for the mapping of functional hippocampal connectivity within the SBF timing model are illustrated in Figure 2.
Further understanding of the hippocampus's role in interval timing could be achieved by examining the differences between pre- and post-hippocampal lesion training on a single-trial level (Church et al., 1994). This would allow us to narrow the range of possible roles the hippocampus

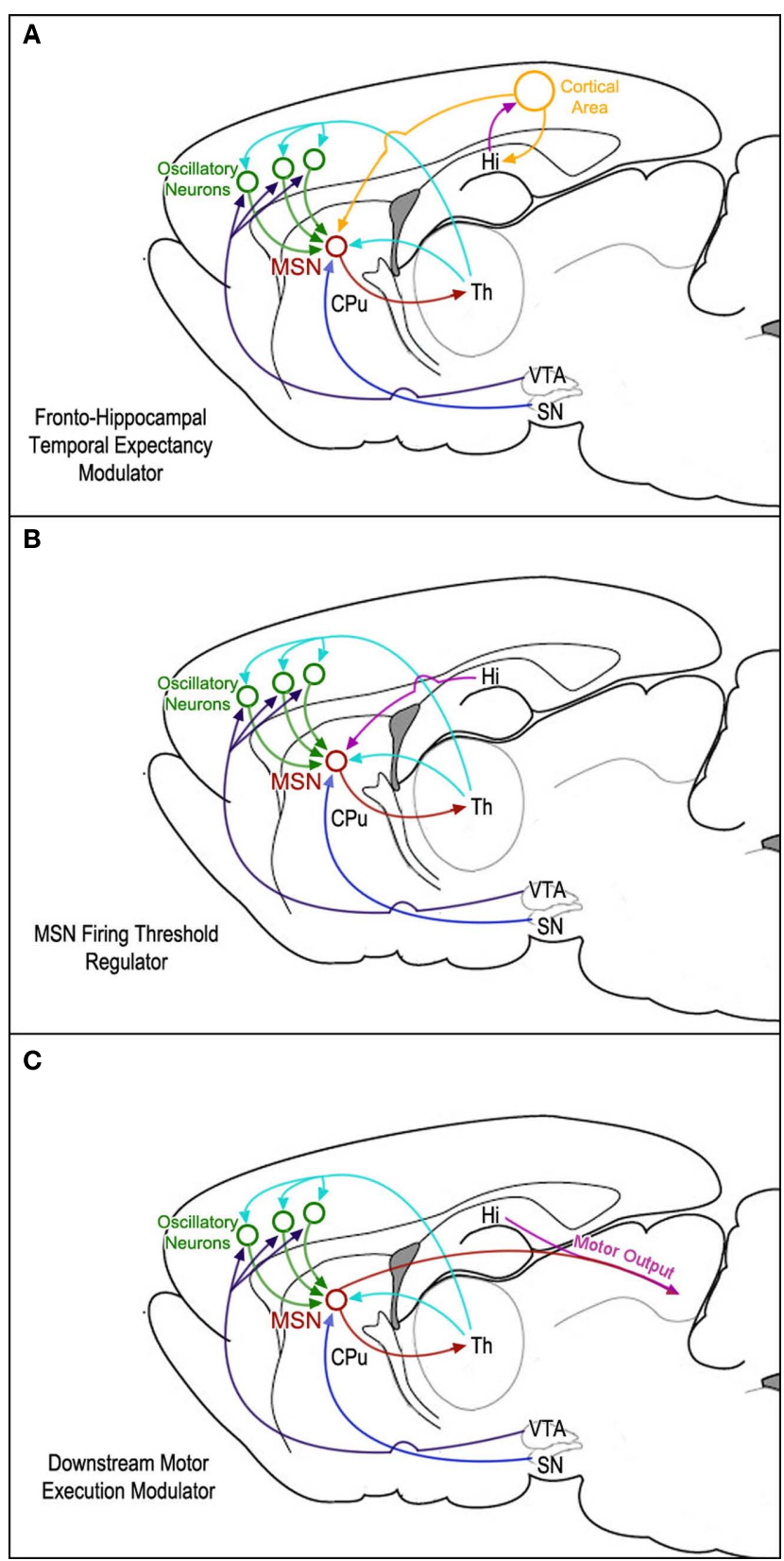

FIGURE 2 | Diagrams of three possible mappings of functional hippocampal connectivity within the neural circuits proposed by the striatal beat-frequency (SBF) model of interval timing: (A) the hippocampus is involved in a feedback mechanism designed to update temporal expectancy with a separate cortical area. This area's output is then integrated with clock and reward information by striatal medium spiny neurons (MSNs). (B) The hippocampus modulates MSN firing thresholds via either tonic inhibition or phasic excitation. (C) Hippocampal regulation downstream of the MSNs affects translation of temporal information into motor output.

might play in either attention, feedback, or memory consolidation mechanisms on a trial-by-trial basis (Meck, 1988; Buhusi and Meck, 2002; Buhusi et al., 2003, 2004). It could also provide us with clues as to whether or not the "clock stage" itself is affected, which would be reflected by a proportional horizontal shift of the response states (see Church et al., 1994; Matell et al., 2006, and MacDonald et al., 2011). Conversely, if the horizontal (e.g., leftward) shift in timing functions resulting from hippocampal damage is due to a change (e.g., decrease) in the latency to start timing rather than in the centering of the distribution of responses around the target duration, then it might suggest the third possibility discussed above. Furthermore, in order to examine the interaction between the hippocampus and either the cortex or the striatum, one could employ a crosslesioning technique wherein one of each structure would be compromised contralaterally in addition to a transection of the corpus callosum (e.g., Christakou et al., 2001; Chudasama et al., 2003). Moreover, future studies would benefit from the use of optogenetic techniques (Yizhar et al., 2011) in terms of identifying the functional "connectome" among the hippocampus, striatum, and cortex (Chuhma et al., 2011). This would provide regions of interest for more traditional electrophysiological and pharmacological mapping studies of the role of the hippocampus and other brain structures in time - the fourth dimension of neural function (Coull et al., 2011).

\section{REFERENCES}

Allman, M. J., and Meck, W.H. (2011). Pathophysiological distortions in time perception and timed performance. Brain (in press).

Balci, F., Meck, W. H., Moore, H., and Brunner, D. (2009). "Timing deficits in aging and neuropathology," in Animal Models of Human Cognitive Aging, eds J.L. Bizon and A. Wood (Totowa, NJ: Humana Press), 161-201.

Berke, J. D., Okatan, M., Skurski, J., and Eichenbaum, H. B. (2004). Oscillatory entrainment of striatal neurons in freely moving rats. Neuron $43,883-896$.

Buhusi, C. V., Buhusi, M. C., Scripal, I., Maness, P., Schachner, M., and Meck, W. H. (2003). Impaired attentional control of temporal and spatial information in CHL1 KO mice. Abstr. Soc. Neurosci. 860, 11.

Buhusi, C. V., and Meck, W. H. (2002). Ibotenic lesions of the hippocampus disrupt attentional control of interval timing. Abstr. Soc. Neurosci. 183, 1.

Buhusi, C. V., and Meck, W. H. (2005). What makes us tick? Functional and neural mechanisms of interval timing. Nat. Rev. Neurosci. 6, 755-765.

Buhusi, C. V., Mocanu, M., and Meck, W. H. (2004). Abnormal memory consolidation of intevral timing 
in rats with ibotenic lesions of the hippocampus. Abstr. Soc. Neurosci. 550, 18.

Carter, A. G., Soler-Llavina, G. J., and Sabatini, B. L. (2007). Timing and location of synaptic inputs determine modes of subthreshold integration in striatal medium spiny neurons. J. Neurosci. 27, 8967-8977.

Centonze, D., Picconi, B., Gubellini, P., Bernardi, G., and Calabresi, P. (2001). Dopaminergic control of synaptic plasticity in the dorsal striatum. Eur. J. Neurosci. 13, 1071-1077.

Cheng, R. K., Ali, Y. M., and Meck, W. H. (2007a). Ketamine "unlocks" the reduced clock-speed effects of cocaine following extended training: evidence for dopamine - glutamate interactions in timing and time perception. Neurobiol. Learn. Mem. 88, 149-159.

Cheng, R. K., Hakak, O. L., and Meck, W. H. (2007b). Habit formation and the loss of control of an internal clock: inverse relationship between the level of baseline training and the clock-speed enhancing effects of methamphetamine. Psychopharmacology (Berl.) $193,351-362$.

Cheung,T.H.C., and Cardinal, R.N. (2005). Hippocampal lesions facilitate instrumental learning with delayed reinforcement but induce impulsive choice in rats. BMC Neurosci. 6, 36. doi: 10.1186/1471-2202-6-36

Christakou, A., Robbins, T. W., and Everitt, B. J. (2001). Functional disconnection of a prefrontal corticaldorsal striatal system disrupts choice reaction time performance: implications for attentional function. Behav. Neurosci. 115, 812-825.

Chudasama, Y., Baunez, C., and Robbins, T. W. (2003). Functional disconnection of the medial prefrontal cortex and subthalamic nucleus in attentional performance: evidence for corticosubthalamic interaction. J. Neurosci. 23, 5477-5485.

Chuhma, N., Tanaka, K. F., Hen, R., and Rayport, S. (2011). Functional connectome of the striatal medium spiny neuron. J. Neurosci. 31, 1183-1192.

Church, R. M., Meck, W. H., and Gibbon, J. (1994). Application of scalar timing theory to individual trials. J. Exp. Psychol. Anim. Behav. Process. 20, 135-155.

Coull, J. T., Cheng, R. K., and Meck, W. H. (2011). Neuroanatomical and neurochemical substrates of timing. Neuropsychopharmacology 36, 3-25.

Coull, J. T., Vidal, F., Nazarian, B., and Macar, F. (2004). Functional anatomy of the attentional modulation of time estimation. Science 303, 1506-1508.

Devan, B. D., and White, N. M. (1999). Parallel information processing in the dorsal striatum: relation to hippocampal function. J. Neurosci. 19, 2789-2798.

Dietrich, A., and Allen, J. D. (1998). Functional dissociation of the prefrontal cortex and the hippocampus in timing behavior. Behav. Neurosci. 112, 1043-1047.

Dietrich, A., Allen, J. D., and Bunnell, B. N. (1997). Is the hippocampus involved in temporal discrimination and the memory of short intervals? Int. J. Neurosci. $90,255-269$.

Gibbon, J., Malapani, C., Dale, C. L., and Gallistel, C. R. (1997). Toward a neurobiology of temporal cognition: advances and challenges. Curr. Opin. Neurobiol. 7, 170-184.

Graham, S., Phua, E., Soon, C. S., Oh, T., Au, C., Shuter, B., Wang, S. C., and Yeh, I. B. (2009). Role of medial cortical, hippocampal and striatal interactions during cognitive set-shifting. Neuroimage 45, 1359-1367.

Grossberg, S., and Merrill, J. (1992). A neural network model of adaptively timed reinforcement learning and hippocampal dynamics. Brain Res. Cogn. Brain Res. 1, 3-38.

Grossberg, S., and Merrill, J. (1996). The hippocampus and cerebellum in adaptively timed learning, recognition, and movement. J. Cogn. Neurosci. 8 , 257-277.

Harrington, D. L., Boyd, L. A., Mayer, A. R., Sheltraw, D. M., Lee, R. R., Huang, M., and Rao, S. M. (2004). Neural representation of interval encoding and decision making. Brain Res. Cogn. Brain Res. 21, 193-205.

Harrington, D. L., Castillo, G. N., Fong, C. H., and Reed, J. D. (2011). Neural underpinnings of distortions in the experience of time across senses. Front. Integr. Neurosci. 5:32. doi: 10.3389/fnint.2011.00032

Harrington, D. L., Zimbelman, J. L., Hinton, S. C., and Rao, S. M. (2010). Neural modulation of temporal encoding, maintenance, and decision processes. Cereb. Cortex 20, 1274-1285.

Hinton, S. C., and Meck, W. H. (2004). Frontal-striatal circuitry activated by human peak-interval timing in the supra-seconds range. Brain Res. Cogn. Brain Res. 21, 171-182.

Höhn, S., Dallérac, G., Faure, A., Urbach, Y., Nguyen, H. P., Riess, O., von Hörsten, S., Le Blanc, P., Desvignes, N., El Massioui, N., Brown, B. L., and Doyère, V. (2011). Behavioral and in vivo electrophysiological evidence for presymptomatic alteration of prefronto-striatal processing in the transgenic rat model for Huntington disease. J. Neurosci. 31, 8986-8997.

Jin, D. Z., Fujii, N., and Graybiel, A. M. (2009). Neural representation of time in cortico-basal ganglia circuits. Proc. Natl. Acad. Sci. U.S.A. 106, 19156-19161.

Johnson, A., van der Meer, M. A., and Redish, A.D. (2007). Integrating hippocampus and striatum in decisionmaking. Curr. Opin. Neurobiol. 17, 692-697.

Lee, A. S., Duman, R. S., and Pittenger, C. (2008). A double dissociation revealing bidirectional competition between striatum and hippocampus during learning. Proc. Natl. Acad. Sci. U.S.A. 105, 17163-17168.

Lewis, P.A., Couch, T. J., and Walker, M. P. (2011). Keeping time in your sleep: overnight consolidation of temporal rhythm. Neuropsychologia 49, 115-123.

Lustig, C., Matell, M. S., and Meck, W. H. (2005). Not "just" a coincidence: Frontal-striatal synchronization in working memory and interval timing. Memory 13 , $441-448$.

Lytton, W. W., and Lipton, P. (1999). Can the hippocampus tell time? The temporo-septal engram shift model. Neuroreport 10, 2301-2306.

MacDonald, C. J., Cheng, R. K., and Meck, W. H. (2011). Interval timing and time-based decision making requires differential protein synthesis in the dorsal and ventral striatum for the setting of "start" and "stop" response thresholds. Front. Integr. Neurosci. (in press).

MacDonald, C. J., and Meck, W. H. (2004). Systems-level integration of interval timing and reaction time. Neurosci. Biobehav. Rev. 28, 747-769.

Matell, M. S., Bateson, M., and Meck, W.H. (2006). Singletrials analyses demonstrate that increases in clock speed contribute to the methamphetamine-induced horizontal shifts in peak-interval timing functions. Psychopharmacology (Berl.) 188, 201-212.

Matell,M.S., and Meck,W.H. (2000). Neuropsychological mechanisms of interval timing behavior. Bioessays 22, 94-103.

Matell, M. S., and Meck, W. H. (2004). Cortico-striatal circuits and interval timing: coincidence detection of oscillatory processes. Brain Res. Cogn. Brain Res. 21, 139-170.

Matell, M. S., Meck, W. H., and Nicolelis, M. A. (2003). Interval timing and the encoding of signal duration by ensembles of cortical and striatal neurons. Behav. Neurosci. 117, 760-773.

McHugh, S. B., Campbell, T. G., Taylor, A. M., Rawlins, J. N., and Bannerman, D. M. (2008). A role for dorsal and ventral hippocampus in inter-temporal choice cost-benefit decision making. Behav. Neurosci. 122, $1-8$.

Meck, W. H. (1988). Hippocampal function is required for feedback control of an internal clock's criterion. Behav. Neurosci. 102, 54-60.

Meck, W. H. (1996). Neuropharmacology of timing and time perception. Brain Res. Cogn. Brain Res. 3, 227-242.

Meck, W.H. (2005). Neuropsychology of timing and time perception. Brain Cogn. 58, 1-8.

Meck, W. H. (2006a). Frontal cortex lesions eliminate the clock speed effect of dopaminergic drugs on interval timing. Brain Res. 1108, 157-167.

Meck, W.H. (2006b). Neuroanatomical localization of an internal clock: a functional link between mesolimbic, nigrostriatal, and mesocortical dopaminergic systems. Brain Res. 1109, 93-107.

Meck, W. H., and Benson, A. M. (2002). Dissecting the brain's internal clock: how frontal-striatal circuitry keeps time and shifts attention. Brain Cogn. 48, 195-211.

Meck, W. H., Church, R. M., and Olton, D. S. (1984). Hippocampus, time, and memory. Behav. Neurosci. 98, 3-22.

Meck, W. H., Church, R. M., Wenk, G. L., and Olton, D. S. (1987). Nucleus basalis magnocellularis and medial septal area lesions differentially impair temporal memory. J. Neurosci. 7, 3505-3511.

Meck, W. H., Penney, T. B., and Pouthas, V. (2008). Cortico-striatal representation of time in animals and humans. Curr. Opin. Neurobiol. 18, 145-152.

Melgire, M., Ragot, R., Samson, S., Penney, T. B., Meck, W. H., and Pouthas, V. (2005). Auditory/ visual duration bisection in patients with left or right medial-temporal lobe resection. Brain Cogn. $58,119-124$.

Olton, D. S., Meck, W. H., and Church, R. M. (1987). Separation of hippocampal and amygdaloid involvement in temporal memory dysfunctions. Brain Res. 404, 180-188.

Olton, D. S., Wenk, G. L., Church, R. M., and Meck, W. H. (1988). Attention and the frontal cortex as examined by simultaneous temporal processing. Neuropsychologia 26, 307-318.

Onoda, K., Takahashi, E., and Sakata, S. (2003). Eventrelated potentials in the frontal cortex, hippocampus, and cerebellum during a temporal discrimination task in rats. Brain Res. Cogn. Brain Res. 17, 380-387.

Oprisan, S. A., and Buhusi, C. V. (2011). Modelling pharmacological clock and memory patterns of interval timing in a striatal beat-frequency model with realistic, noisy neurons. Front. Integr. Neurosci. (in press).

Poldrack, R.A., and Packard, M. G. (2003). Competition among multiple memory systems: converging evidence from animal and human brain studies. Neuropsychologia 41, 245-251.

Sakata, S. (2006). Timing and hippocampal theta in animals. Rev. Neurosci. 17, 157-162. 
Sala, M., Caverzasi, E., Lazzaretti, M., Morandotti, N., De Vidovich, G., Marraffini, E., Gambini, F., Isola, M., De Bona, M., Rambaldelli, G., d'Allio, G., Barale, F., Zappoli, F., and Brambilla, P. (2011). Dorsolateral prefrontal cortex and hippocampus sustain impulsivity and aggressiveness in borderline personality disorder. J. Affect. Disord. 131, 417-421.

Stern, E. A., Kincaid, A. E., and Wilson, C. J. (1997). Spontaneous subthreshold membrane potential fluctuations and action potential variability of rat corticostriatal and striatal neurons in vivo. J. Neurophysiol. 77, 1697-1715.

Vidalaki, V. N., Ho, M. Y., Bradshaw, C. M., and Szabadi, E. (1999). Interval timing performance in temporal lobe epilepsy: differences between patients with left and right hemisphere foci. Neuropsychologia 37, 1061-1070.

Wearden, J. H. (2004). Decision processes in models of timing. Acta Neurobiol. Exp. (Wars) 64, 303-317.
Wiltgen, B. J., and Silva, A. J. (2007). Memory for context becomes less specific with time. Learn. Mem. 14, 313-317.

Wiltgen, B. J., Zhou, M., Cai, Y., Balaji, J., Karlsson, M. G., Parivash, S. N., Li, W., and Silva, A. J. (2010). The hippocampus plays a selective role in the retrieval of detailed contextual memories. Curr. Biol. 20, 1336-1344.

Yin, H. H. (2009). The role of the murine motor cortex in action duration and order. Front. Integr. Neurosci. 3:23. doi: 10.3389/neuro.07.023.2009

Yin, H. H., and Knowlton, B. J. (2006). The role of the basal ganglia in habit formation. Nat. Neurosci. Rev. 7, 464-476.

Yin, H. H., Mulcare, S. P., Hilario, M. R., Clouse, E., Holloway, T., Davis, M. I., Hansson, A. C., Lovinger, D. M., and Costa, R. M. (2009). Dynamic reorganization of striatal circuits during the acquisition and consolidation of a skill. Nat. Neurosci. 12, 333-341.

Yizhar, O., Fenno, L. E., Davidson, T. J., Mogri, M., and Deisseroth, K. (2011). Optogenetics in neural systems. Neuron 71, 9-34.

Received: 26 July 2011; accepted: 29 July 2011; published online: 11 August 2011.

Citation: Yin B and Troger AB (2011) Exploring the 4th dimension: hippocampus, time, and memory revisited. Front. Integr. Neurosci. 5:36. doi: 10.3389/ fnint.2011.00036

Copyright (C) 2011 Yin and Troger. This is an open-access article subject to a non-exclusive license between the authors and Frontiers Media SA, which permits use, distribution and reproduction in other forums, provided the original authors and source are credited and other Frontiers conditions are complied with. 